\title{
OPEN Menopause impacts human brain structure, connectivity, energy metabolism, and amyloid-beta deposition
}

\author{
Lisa Mosconi ${ }^{1,2,3 凶}$, Valentina Berti ${ }^{4}$, Jonathan Dyke ${ }^{2}$, Eva Schelbaum ${ }^{1}$, Steven Jett ${ }^{1}$, \\ Lacey Loughlin ${ }^{1}$, Grace Jang ${ }^{1}$, Aneela Rahman ${ }^{1}$, Hollie Hristov ${ }^{1}$, Silky Pahlajani ${ }^{1}{ }^{2}$, \\ Randolph Andrews ${ }^{5}$, Dawn Matthews ${ }^{5}$, Orli Etingin ${ }^{6}$, Christine Ganzer ${ }^{7}$, Mony de Leon ${ }^{2}$, \\ Richard Isaacson ${ }^{1} \&$ Roberta Diaz Brinton ${ }^{8}$
}

\begin{abstract}
All women undergo the menopause transition (MT), a neuro-endocrinological process that impacts aging trajectories of multiple organ systems including brain. The MT occurs over time and is characterized by clinically defined stages with specific neurological symptoms. Yet, little is known of how this process impacts the human brain. This multi-modality neuroimaging study indicates substantial differences in brain structure, connectivity, and energy metabolism across MT stages (pre-menopause, peri-menopause, and post-menopause). These effects involved brain regions subserving higher-order cognitive processes and were specific to menopausal endocrine aging rather than chronological aging, as determined by comparison to age-matched males. Brain biomarkers largely stabilized post-menopause, and gray matter volume (GMV) recovered in key brain regions for cognitive aging. Notably, GMV recovery and in vivo brain mitochondria ATP production correlated with preservation of cognitive performance post-menopause, suggesting adaptive compensatory processes. In parallel to the adaptive process, amyloid- $\beta$ deposition was more pronounced in perimenopausal and post-menopausal women carrying apolipoprotein E-4 (APOE-4) genotype, the major genetic risk factor for late-onset Alzheimer's disease, relative to genotype-matched males. These data show that human menopause is a dynamic neurological transition that significantly impacts brain structure, connectivity, and metabolic profile during midlife endocrine aging of the female brain.
\end{abstract}

The menopause transition (MT) is a midlife neuroendocrine aging process specific to females that culminates with reproductive senescence ${ }^{1}$. All women undergo menopause in their lifetime either through the natural endocrine aging process or through medical intervention. The MT occurs in stages characterized by unique endocrine properties that impact aging trajectories of multiple organ systems including brain ${ }^{1}$. While menopause is a reproductive transition state, it is also a neurological transition ${ }^{1}$, as evidence by the fact that many menopausal symptoms are neurological in nature, such as hot flashes, disturbed sleep, mood changes, and forgetfulness ${ }^{2}$.

The MT is orchestrated by gonadal sex steroid hormones, which are known regulators of both reproductive and neural function ${ }^{1}$. During the MT, sex hormones, especially $17 \beta$-estradiol, substantially decline in body and brain $^{3}$. Molecular, cellular and systems biology characterization of the MT in brain demonstrate a broad impact of estrogen declines on neural processes via genomic and non-genomic actions ${ }^{1}$, including changes in spinogenesis and synaptogenesis, neuronal number, morphology, glucose metabolic rates, and gene expression ${ }^{1,3}$. Additionally, estrogen depletion has been linked to amyloid-beta $(A \beta)$ plaque accumulation, a hallmark of Alzheimer's disease (AD), in female animals ${ }^{4,5}$. However, little is known of how the MT impacts the human brain.

\footnotetext{
${ }^{1}$ Department of Neurology, Weill Cornell Medicine, 420 East 70th, LH-404, New York, NY 10021, USA. ${ }^{2}$ Department of Radiology, Weill Cornell Medical College, New York, NY, USA. ${ }^{3}$ Department of Psychiatry, New York University School of Medicine, New York, NY, USA. "Department of Nuclear Medicine, University of Florence, Florence, Italy. ${ }^{5}$ ADM Diagnostics, Chicago, IL, USA. ${ }^{6}$ Department of Internal Medicine, Weill Cornell Medical College, New York, NY, USA. ${ }^{7}$ Hunter-Bellevue School of Nursing, Hunter College, CUNY, New York, NY, USA. ${ }^{8}$ Departments of Pharmacology and Neurology, College of Medicine, University of Arizona, Tucson, AZ, USA. ${ }^{\square}$ email: lim2035@ med.cornell.edu
} 


\begin{tabular}{|c|c|c|c|}
\hline & Pre-menopausal group & Peri-menopausal group & Post-menopausal group \\
\hline \multicolumn{4}{|c|}{ Clinical and demographic measures } \\
\hline $\mathrm{N}$ & 30 & 57 & 74 \\
\hline Age, years, range & $44(4), 40-53$ & $50(4), 41-60$ & $57(4)^{*}, 46-65$ \\
\hline Education, years & $17(3)$ & $17(2)$ & $17(2)$ \\
\hline Ethnicity, \% White & 80 & 77 & 89 \\
\hline MMSE score & $29(1)$ & $29(1)$ & $29(1)$ \\
\hline APOE $\varepsilon 4$ carriers, $\%$ positive & 43 & 33 & 48 \\
\hline Hysterectomy status, \% positive & 0 & 6 & 19 \\
\hline \multicolumn{4}{|l|}{ Menopausal hormonal therapy } \\
\hline$\%$ Current users & 0 & 13 & 32 \\
\hline$\%$ Past users & 0 & 2 & 4 \\
\hline \multicolumn{4}{|l|}{ Cognitive measures } \\
\hline Global cognition, mean (SE) & $-0.11(0.17)$ & $-0.02(0.09)$ & $0.06(0.11)$ \\
\hline Adjusted by APOE-4 status & $-0.09(0.17)$ & $-0.03(0.09)$ & $0.06(0.11)$ \\
\hline Memory, mean (SE) & $0.05(0.20)$ & $0.06(0.11)$ & $0.02(0.13)$ \\
\hline Adjusted by APOE-4 status & $0.07(0.21)$ & $0.04(0.11)$ & $0.02(0.13)$ \\
\hline
\end{tabular}

Table 1. Participants' characteristics by menopause status. Clinical measures are means (SD), unless otherwise specified. Cognitive measures are age and education-adjusted means (SE). ${ }^{\star}$ Different from PRE, $p<0.05$.

Brain imaging analyses conducted in post-menopausal women first reported declines in glucose metabolism and gray matter volume (GMV), which were modulated by menopause hormone treatment $(\mathrm{HT})^{6,7}$, indicating that neuro-endocrine processes retain dynamic properties well into menopause. However, data in women undergoing the MT are scarce. We previously reported that, among those with genetic risk factors for AD such as apolipoprotein epsilon-4 genotype (APOE- 4$)^{8}$, the MT was associated with lower GMV, hypometabolism, and emerging $A \beta$ deposition ${ }^{9-11}$. It is unknown whether similar changes occur in women without genetic risks for $\mathrm{AD}$, or whether the MT impacts other structural, bioenergetic and cognitive indicators.

Herein, we conducted a multi-modality neuroimaging study of women at different MT stages (pre-, peri-, and post-menopause) to investigate MT's effects on brain's gray (GM) and white matter (WM) structure, connectivity, energy metabolism, and A $\beta$ deposition. Results indicate that the MT significantly impacts all these brain biomarkers in regions involved in higher-order cognitive functions. Effects were independent of age and HT use, and were specific to menopausal endocrine aging rather than chronological aging, as determined by comparison with age-matched males. Notably, cognition was preserved post-menopause, which correlated with GMV recovery and brain adenosine triphosphate (ATP) production, highlighting potential compensatory mechanisms. Finally, A $\beta$ deposition was greater in post-menopausal and peri-menopausal women carrying APOE- 4 genotype, indicating APOE-4 specific effects on AD risk with onset in the peri-menopause.

\section{Results}

Participants. A total of 182 cognitively intact $40-65$ year-old women were enrolled. Nine participants were excluded due to neuropathological conditions encountered in the MR images ( $n=7$; e.g. neoplastic condition or aneurysm), or due to artifacts $(\mathrm{n}=2)$. Three participants with incomplete clinical data and 9 with unclear menopause status were also excluded. Our final sample consisted of 161 women, including 30 pre-menopausal (PRE), 57 peri-menopausal (PERI), and 74 post-menopausal (POST) participants.

Participants' characteristics are found in Table 1. There were no group differences in demographic and clinical measures except for an age difference between PRE and POST groups. As described in "Methods", our procedures to address age effects included (i) adding age as a confounder in all analyses, and (ii) comparing each MT group to an age-matched male group: consisting of 30 males age-matched to the PRE group (MALE $\mathrm{PRE}_{\text {) }}$, 50 age-matched

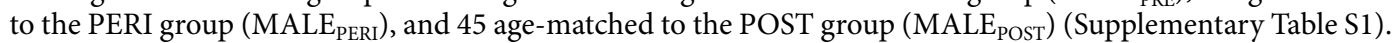
Forty-two percent of participants were APOE-4 positive, with comparable distributions between groups.

Biomarker results. We examined a panel of brain biomarkers examining:

- Structure: GMV and white matter volume (WMV) via Magnetic Resonance Imaging (MRI), and fractional anisotropy (FA, an index of WM integrity and structural connectivity ${ }^{12}$ ) via MRI-Diffusion Tensor Imaging (DTI);

- Energy metabolism: glucose metabolism (CMRglc) via ${ }^{18} \mathrm{~F}$-fluorodeoxy-2-glucose (FDG) Positron Emission tomography (PET), cerebral blood flow (CBF) via Arterial Spin Labeling (ASL), and ATP production via ${ }^{31}$ Phosphorus-Magnetic Resonance Spectroscopy $\left({ }^{31} \mathrm{P}-\mathrm{MRS}\right)$;

- $\mathrm{A} \beta$ deposition via ${ }^{11} \mathrm{C}$-Pittsburgh compound $\mathrm{B}(\mathrm{PiB}) \mathrm{PET}$. 
Unless otherwise specified, all below voxel-based results are significant at $p<0.05$, cluster-level corrected for family-wise type error (FWE), adjusted for age, APOE-4 status, and modality-specific confounders.

Biomarker differences between MT groups. Structural biomarkers. GMV. MT stage effects were observed in inferior temporal gyrus, precuneus, and fusiform gyrus of the right hemisphere (Fig. 1a). On posthoc analysis, the temporal cluster reflected lower GMV in the POST group compared to the PRE group, while the precuneus and fusiform clusters reflected lower GMV in the PERI group compared to the POST group (Fig. 1b, and Supplementary Table S2).

WMV. MT stage effects were observed in anterior and posterior corona radiata, which reflected lower WMV in POST versus PRE and PERI groups (Fig. 1c, d, and Supplementary Table S2).

FA. The only cluster showing MT stage effects that survived correction for multiple comparisons was the right external capsule. In this cluster, the POST group had lower FA than PERI (cluster extent 71 voxels, $x, y, z=33$, $-9,-6, Z=3.81, p=0.017$; Fig. 1e, f). There was no anatomical overlap between FA and WMV maps, as shown in Fig. $1 \mathrm{~g}$.

Metabolic biomarkers. CMRglc. MT stage effects were observed in supramarginal gyri, middle and inferior temporal gyri (Fig. 2a). On post-hoc analysis, all clusters reflected lower CMRglc in the POST group compared to the PRE and PERI groups, and lower CMRglc in right middle temporal gyrus of the PERI compared to the PRE group (Fig. 2b, and Supplementary Table S3).

CBF. MT stage effects were observed in supramarginal gyrus, middle and superior temporal gyrus, superior and inferior frontal gyrus of both hemispheres, which on post-hoc analysis, reflected higher CBF in POST versus PERI (Fig. 2c, d, and Supplementary Table S3).

Figure 2e displays the regional overlap of CMRglc and CBF effects.

ATP production. We examined regional ATP to phosphocreatine (PCr) ratios in parieto-temporal regions showing MT-stage effects on CMRglc and CBF (Table 2). Multivariate general linear models (mGLM) showed higher ATP/PCr in temporal regions $(p$ 's $\leq 0.047)$ and borderline higher ATP/PCr in parietal regions $(p<0.12)$ of POST versus PRE groups. Results remained significant adjusting by APOE-4 status (Table 2).

Amyloid- $\beta$ load. Results are reported in Table 2. Adjusting by age and cerebellar uptake, there were no significant differences in PiB uptake between MT groups. However, adding APOE- 4 status as a covariate enhanced group differences, resulting in higher $\mathrm{PiB}$ uptake in POST versus PRE $(27 \%, p=0.006)$, and PERI versus PRE groups $(18 \%, p=0.056)$. PiB differences between PERI and PRE groups were more pronounced among APOE-4 carriers (Table 2).

Comparisons to age-matched males. To determine whether MT-stage effects on biomarkers were specific for menopause endocrine aging versus chronological aging, we compared each MT group to an agematched male group. We first tested for group biomarker differences within the brain regions showing the MT group-specific effects reported above. We then tested for group differences in the entire search volume.

Structural biomarkers. GMV. Results are presented in Fig. 3a, and Supplementary Table S4. Examination of brain regions showing MT-stage effects indicated lower GMV in inferior temporal gyrus of the POST group compared to MALE $\mathrm{POST}_{\text {, }}$ and lower GMV in precuneus and fusiform gyrus of the PERI group compared to MALE $_{\text {PERI }}$. Across the entire search volume, both POST and PERI groups exhibited additional widespread areas of lower GMV compared to males, involving middle and medial temporal (MTL) regions, superior, middle, and orbital frontal gyrus, anterior cingulate (ACC), insula, and putamen. The PRE group showed lower GMV versus $\mathrm{MALE}_{\mathrm{PRE}}$ in MTL, frontal gyrus, putamen, and inferior and middle temporal cortex.

WMV. Results are presented in Fig. 3b, and Supplementary Table S5. In examination of brain regions exhibiting MT-stage effects, lower WMV in corona radiata of the POST group compared to MALE $\mathrm{POST}_{\text {was }}$ evident. In the entire search volume, the POST group exhibited additional areas of lower WMV in several tracts including superior longitudinal fasciculus (SLF), posterior thalamic radiation, and cortico-spinal tracts. The PERI group exhibited a similar pattern of lower WMV compared to MALE $_{\text {PERI }}$, and the PRE group showed lower WMV in internal capsule compared to MALE $\mathrm{PRE}_{\text {. }}$

FA. Results are presented in Fig. 3c, and Supplementary Table S6. Examination of brain regions showing MTstage effects revealed higher FA in corona radiata and fornix in the POST group compared to MALE $\mathrm{POST}_{\text {. There }}$

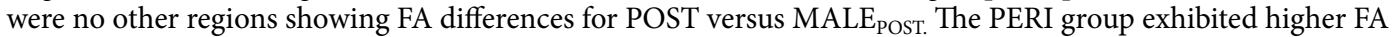
in corona radiata, fornix, external capsule and uncinate fasciculus; and lower FA in SLF and posterior thalamic

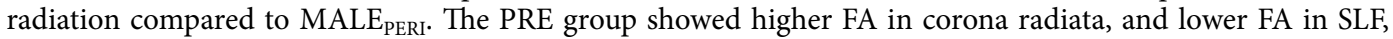
compared to MALE PRE. 
a
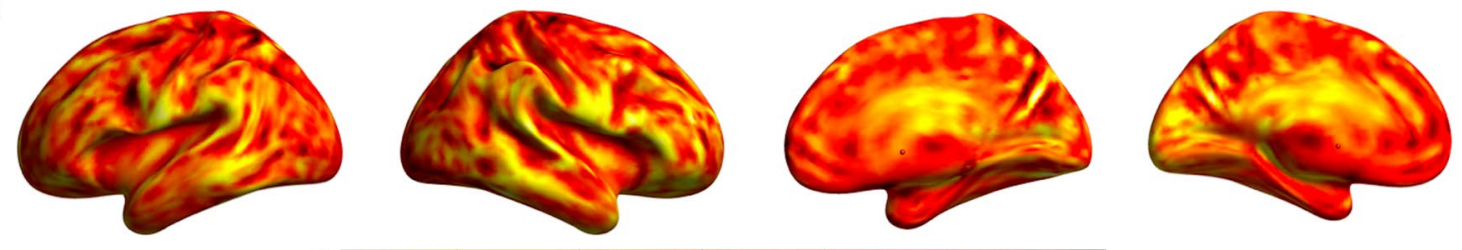

$P$

$<0.001$

$<0.01$

b

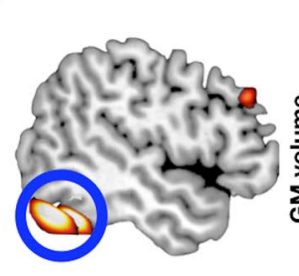

Inferior temporal gyrus

Precuneus

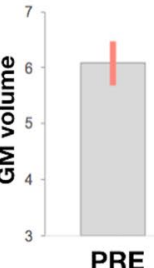

PRE

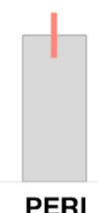

PERI

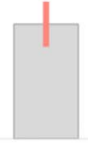

POST
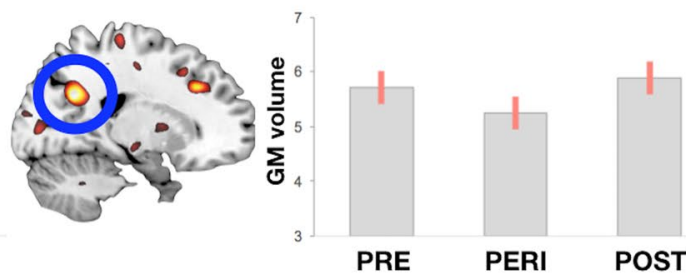

C
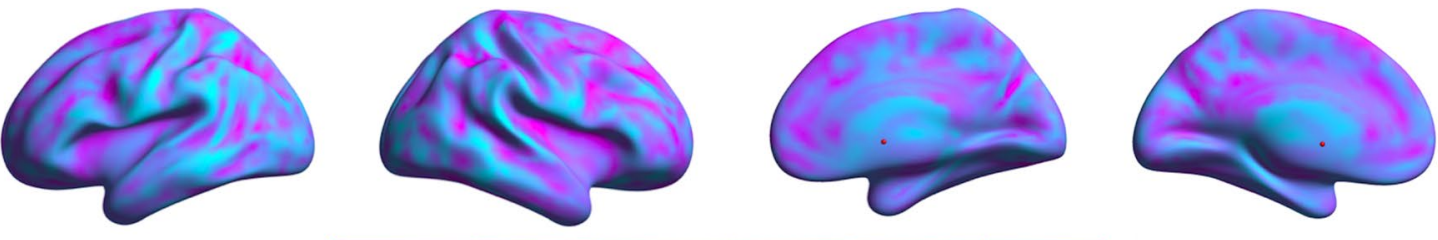

$P$

$<0.001$

$<0.01$

d
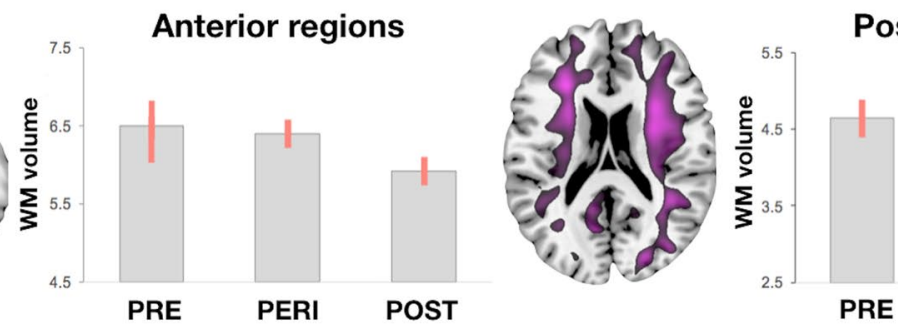

Posterior regions

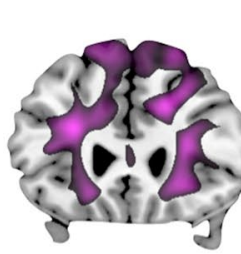

PRE

PERI

POST
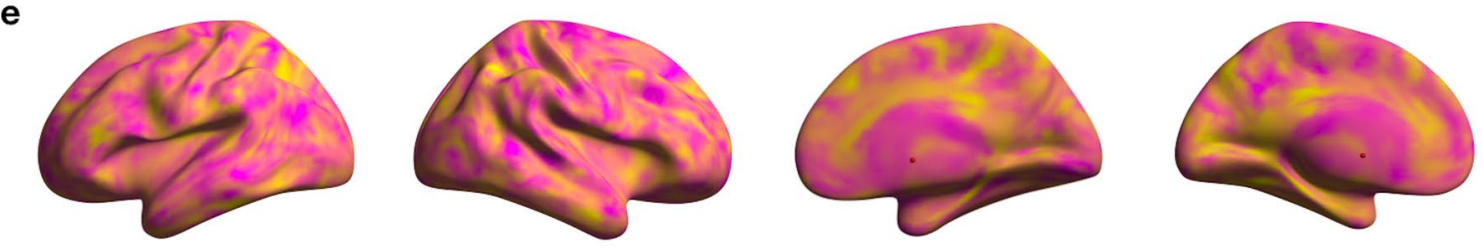

$\boldsymbol{P}$

$<0.001$

$<0.01$

f

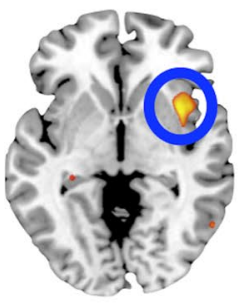

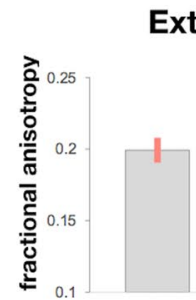

PRE
External capsule

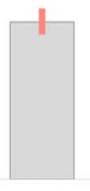

PERI

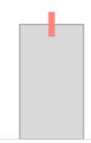

POST g

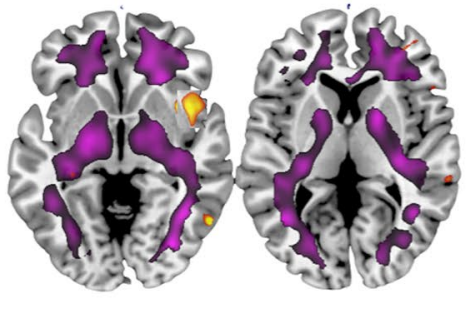

Z score

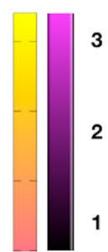

Figure 1. Structural biomarker differences between menopausal groups. (a) Surface maps of regional GMV differences. (b) MRI slice overlays and plots representing GMV in temporal and precuneus clusters. (c) Surface maps of regional WMV differences. (d) MRI slice overlays and plots representing WMV in anterior and posterior areas averaged between hemispheres. (e) Surface maps of regional FA differences. (f) MRI slice overlays and plots representing FA in external capsule. (g) Slice overlays depicting lack of overlap between WMV (purple) and FA (yellow) effects. In (a, c, e), SPMs are represented on modality-specific color-coded scales with corresponding $\mathrm{P}$ values. In (g), $\mathrm{Z}$ scores are reported in lieu of $p$ values to enable multi-modality comparison. In (b, d), values are mean (SE). Results are adjusted by age and total intracranial volume. Abbreviations FA, fractional anisotropy; GMV, gray matter volume; PERI, perimenopause; POST, post-menopause; PRE, pre-menopause; SPMs, statistical parametric maps; WMV, white matter volume. 
a
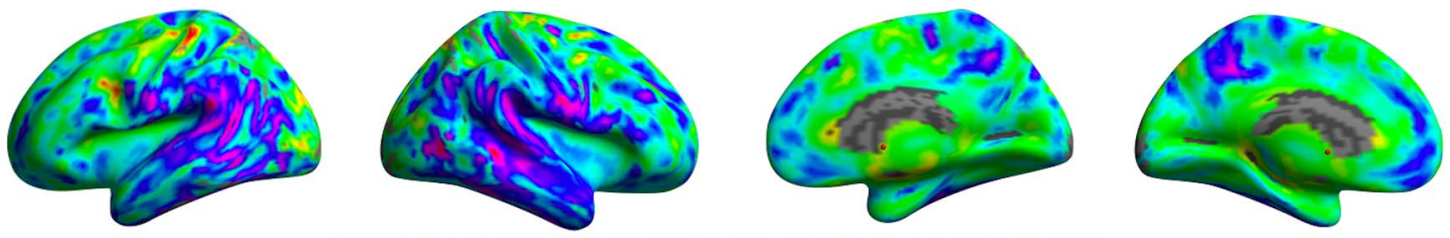

$P$

$<0.01$

b

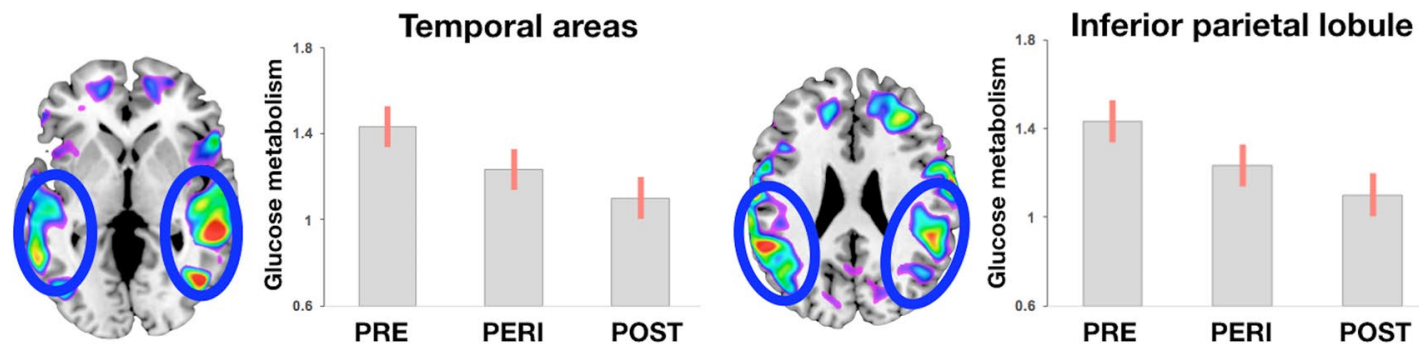

c
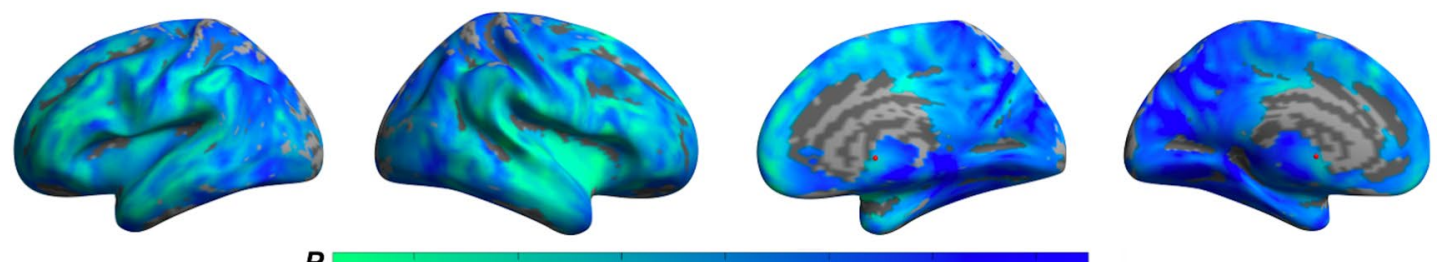

$P$

$<0.01$

d
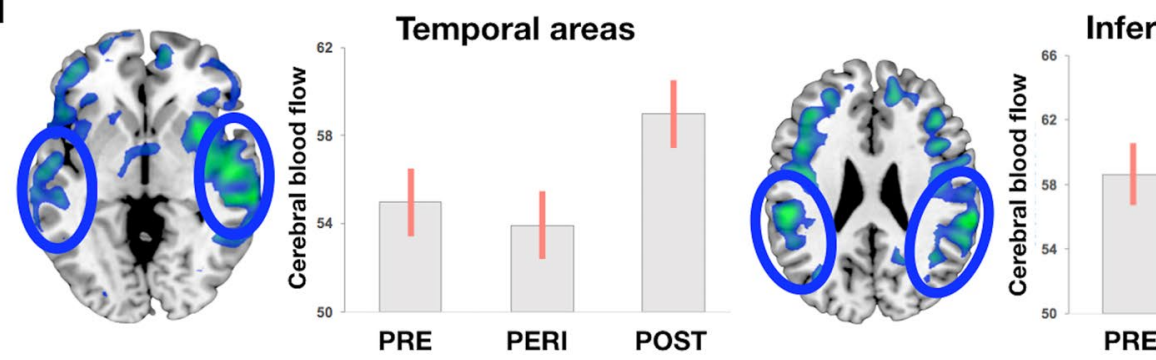

Inferior parietal lobule

e

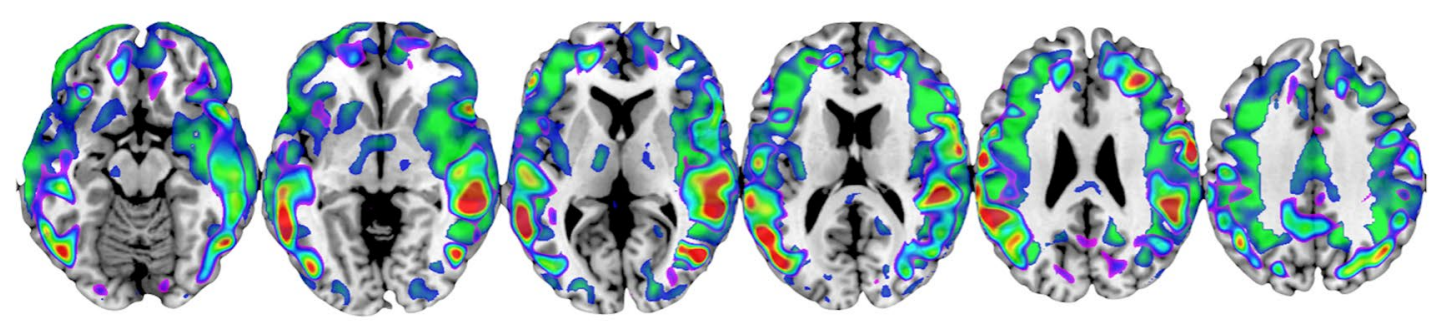

Z score

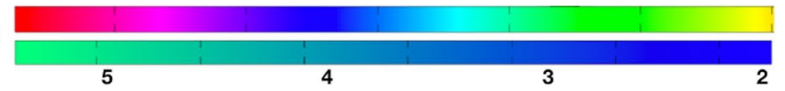

Figure 2. Metabolic biomarker differences between menopausal groups. (a) Surface maps of regional CMRglc differences. (b) MRI slice overlays and plots representing CMRglc in temporal and parietal regions. (c) Surface maps of regional CBF differences. (d) MRI slice overlays and plots representing CBF extracted in temporal and parietal clusters. (e) Slice overlays depicting the overlap between CMRglc (green to red scale) and CBF (blue to green scale) effects. (f) In (a, c), SPMs are represented on modality-specific color-coded scales with corresponding $p$ values. In (e), Z scores are reported to enable multi-modality comparisons. In (b, d), values are mean (SE). Results are adjusted by age and global activity. Abbreviations CBF, cerebral blood flow; CMRglc, cerebral glucose metabolism; PERI, peri-menopause; POST, post-menopause; PRE, pre-menopause; SPMs, statistical parametric maps. 


\begin{tabular}{|c|c|c|c|}
\hline & PRE & PERI & POST \\
\hline \multicolumn{4}{|l|}{ ATP/PCr measures } \\
\hline Superior temporal gyrus & $1.11(0.05)$ & $1.18(0.03)$ & $1.26(0.03)^{*}$ \\
\hline Adjusted by APOE status & $1.14(0.04)$ & $1.18(0.03)$ & $1.24(0.02)^{*}$ \\
\hline Middle temporal gyrus & $1.09(0.04)$ & $1.13(0.03)$ & $1.20(0.02)^{*}$ \\
\hline Adjusted by APOE status & $1.10(0.03)$ & $1.14(0.02)$ & $1.19(0.02)^{*}$ \\
\hline Inferior temporal gyrus & $1.07(0.04)$ & $1.11(0.02)$ & $1.13(0.02)^{*}$ \\
\hline Adjusted by APOE status & $1.07(0.03)$ & $1.10(0.02)$ & $1.14(0.02)^{*}$ \\
\hline Inferior parietal lobule & $0.77(0.06)$ & $0.76(0.05)$ & $0.83(0.04)$ \\
\hline Adjusted by APOE status & $0.78(0.04)$ & $0.75(0.03)$ & $0.83(0.03)^{*}$ \\
\hline \multicolumn{4}{|l|}{ Amyloid- $\beta$ load } \\
\hline AD-mask SUVR & $1.01(0.12)$ & $1.20(0.06)$ & $1.27(0.07)$ \\
\hline Adjusted by APOE status & $1.00(0.09)$ & $1.21(0.06)$ & $1.29(0.06)^{*}$ \\
\hline \multicolumn{4}{|c|}{ AD-mask SUVR by APOE-4 status } \\
\hline APOE-4- & $0.94(0.12)$ & $1.14(0.07)$ & $1.32(0.07)^{*}$ \\
\hline APOE-4+ & $1.07(0.12)$ & $1.32(0.11)^{*}$ & $1.25(0.08)^{\star}$ \\
\hline
\end{tabular}

Table 2. Region-of-Interest (ROI) measures by menopausal stage group. APOE-4-, APOE-4 non-carriers; APOE-4+, APOE-4 carriers; PERI, peri-menopausal group; POST, post-menopausal group; PRE, premenopausal group; SUVR, standardized uptake value ratio to cerebellar gray matter PiB uptake. Values are means (SE), unless otherwise specified. ${ }^{\star}$ Different from PRE, $p<0.05$.

POST vs MEN

a

b
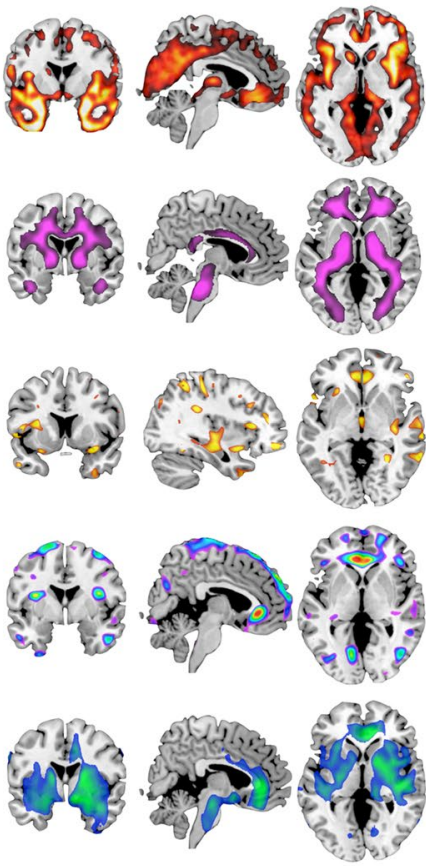

PERI vs MEN
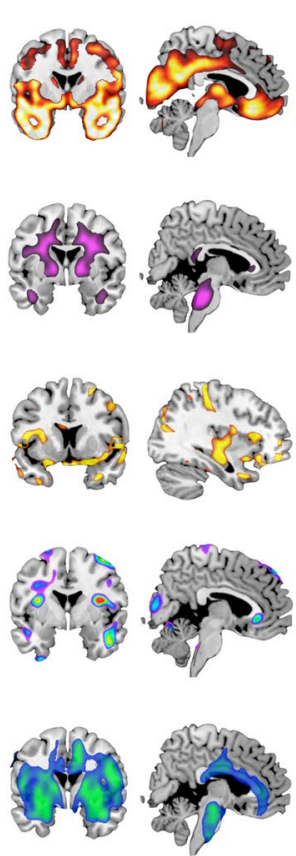
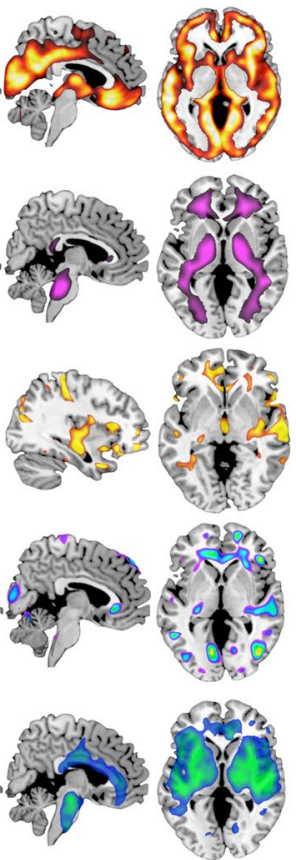

PRE vs MEN
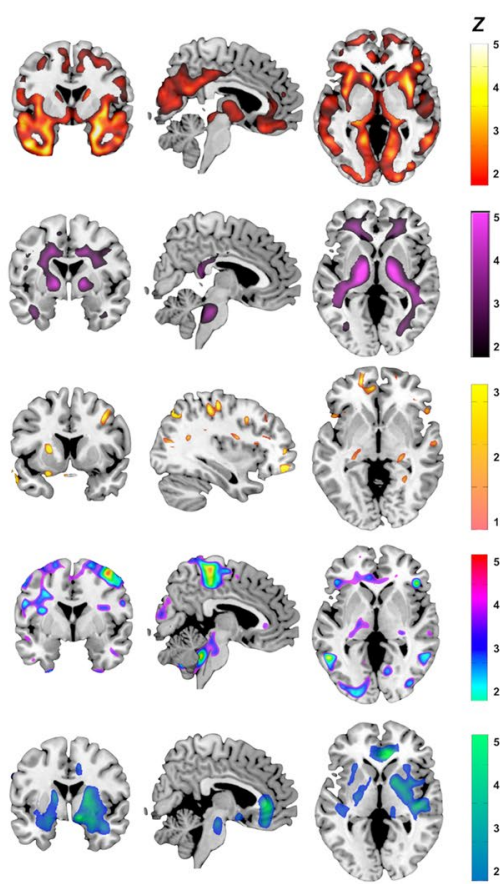

Figure 3. MRI slice overlays displaying biomarker differences between each MT group and males in the corresponding age ranges: (a) Lower GMV in (left) POST, (middle) PERI, and (right) PRE groups versus males. (b) Lower WMV in (left) POST, (middle) PERI, and (right) PRE groups versus males. (c) Higher FA in (left) POST, and (middle) PERI versus males; (right) no differences between PRE and males. (d) Lower CMRglc in (left) POST, (middle) PERI, and (right) PRE groups versus males. (e) Higher CBF in (left) POST, (middle) PERI, and (right) PRE groups versus males. (f) SPMs are represented on modality-specific color-coded scales with corresponding Z scores to enable multi-modality comparisons. Abbreviations See legend to Figs. 1 and 2.

Metabolic biomarkers. CMRglc. Results are presented in Fig. 3d, and Supplementary Table S7. Examination of brain regions showing MT-stage effects showed lower CMRglc in temporo-parietal areas of the POST group

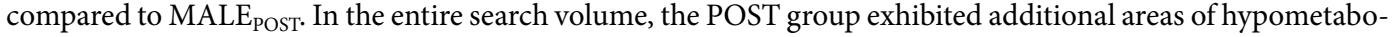
lism in superior and middle frontal cortex compared to males. The PERI group showed lower CMRglc in frontal 


\begin{tabular}{|c|c|c|c|c|c|c|}
\hline & PRE & MALE $_{\text {PRE }}$ & PERI & MALE $_{\text {PERI }}$ & POST & MALE $_{\text {POST }}$ \\
\hline \multicolumn{7}{|l|}{ ATP/PCr measures } \\
\hline Anterior cingulate cortex & $1.06(0.07)$ & $1.11(0.07)$ & $1.10(0.04)$ & $1.12(0.05)$ & $1.12(0.04)$ & $1.12(0.05)$ \\
\hline Adjusted by APOE-4 status & $1.05(0.07)$ & $1.12(0.07)$ & $1.11(0.04)$ & $1.10(0.05)$ & $1.11(0.04)$ & $1.13(0.05)$ \\
\hline Insula & $1.21(0.06)$ & $1.29(0.07)$ & $1.24(0.04)$ & $1.24(0.04)$ & $1.26(0.03)^{*}$ & $1.21(0.05)$ \\
\hline Adjusted by APOE-4 status & $1.20(0.06)$ & $1.30(0.06)$ & $1.24(0.04)$ & $1.23(0.04)$ & $1.27(0.03)^{*}$ & $1.22(0.05)$ \\
\hline Inferior parietal lobule & $0.78(0.07)$ & $0.85(0.08)$ & $0.76(0.04)$ & $0.84(0.04)$ & $0.83(0.03)$ & $0.86(0.04)$ \\
\hline Adjusted by APOE-4 status & $0.77(0.08)$ & $0.86(0.08)$ & $0.76(0.04)$ & $0.84(0.04)$ & $0.83(0.03)$ & $0.86(0.04)$ \\
\hline Middle temporal gyrus & $1.10(0.04)$ & $1.17(0.05)$ & $1.15(0.02)$ & $1.12(0.3)$ & $1.19(0.02)^{*}$ & $1.10(0.03)$ \\
\hline Adjusted by APOE-4 status & $1.10(0.04)$ & $1.18(0.04)$ & $1.15(0.02)$ & $1.12(0.3)$ & $1.19(0.02)^{\star}$ & $1.10(0.03)$ \\
\hline Medial temporal lobe & $1.16(0.05)$ & $1.24(0.05)$ & $1.22(0.03)$ & $1.20(0.04)$ & $1.21(0.03)$ & $1.19(0.04)$ \\
\hline Adjusted by APOE-4 status & $1.15(0.05)$ & $1.26(0.05)$ & $1.22(0.03)$ & $1.20(0.04)$ & $1.22(0.03)$ & $1.19(0.04)$ \\
\hline Medial frontal gyrus & $1.05(0.06)$ & $0.99(0.06)$ & $1.04(0.03)$ & $0.98(0.04)$ & $1.07(0.03)^{*}$ & $0.95(0.04)$ \\
\hline Adjusted by APOE-4 status & $1.04(0.06)$ & $1.01(0.06)$ & $1.05(0.03)$ & $0.97(0.04)$ & $1.07(0.03)^{*}$ & $0.96(0.04)$ \\
\hline Superior frontal gyrus & $0.95(0.05)$ & $0.86(0.06)$ & $0.94(0.03)^{*}$ & $0.84(0.03)$ & $0.96(0.03)^{*}$ & $0.83(0.04)$ \\
\hline Adjusted by APOE-4 status & $0.94(0.05)$ & $0.87(0.05)$ & $0.95(0.03)^{*}$ & $0.84(0.04)$ & $0.96(0.03)^{*}$ & $0.84(0.04)$ \\
\hline \multicolumn{7}{|l|}{ Amyloid- $\boldsymbol{\beta}$ load } \\
\hline AD-mask SUVR & $1.01(0.05)$ & $0.92(0.05)$ & $1.20(0.05)^{*}$ & $0.92(0.06)$ & $1.29(0.05)^{*}$ & $0.93(0.06)$ \\
\hline Adjusted by APOE-4 status & $1.01(0.05)$ & $0.92(0.05)$ & $1.21(0.05)^{\star}$ & $0.91(0.06)$ & $1.29(0.05)^{*}$ & $0.93(0.06)$ \\
\hline \multicolumn{7}{|c|}{ AD-mask SUVR by APOE- 4 status } \\
\hline APOE-4- & $0.94(0.07)$ & $0.86(0.07)$ & $1.14(0.06)$ & $0.88(0.08)$ & $1.32(0.07)^{*}$ & $0.93(0.09)$ \\
\hline APOE-4+ & $1.07(0.07)$ & $0.96(0.08)$ & $1.32(0.09)^{*}$ & $0.93(0.10)$ & $1.25(0.05)^{*}$ & $0.95(0.10)$ \\
\hline
\end{tabular}

Table 3. Regions-of-interest (ROI) measures by age-matched male group comparisons. MALE $\mathrm{PRE}_{\text {, }}$ males age-matched to PRE; MALE $_{\text {PERI }}$, males age-matched to PERI; MALE ${ }_{\mathrm{POST}}$, males age-matched to POST; PERI, peri-menopausal group; POST, post-menopausal group; PRE, pre-menopausal group. Values are means (SE). ${ }^{\star}$ Different from age-matched males, $p<0.05$.

cortex and insula versus MALE $_{\mathrm{PERI}}$, and the PRE group showed lower CMRglc in left supramarginal gyrus versus MALE $_{\text {PRE. }}$.

CBF. Results are presented in Fig. 3e, and Supplementary Table S8. Examination of brain regions exhibiting MT-stage effects showed elevated CBF in frontal regions of POST versus MALE ${ }_{\text {POST }}$. In the entire search volume, the POST group also exhibited lower CBF in ACC, MTL, and basal ganglia compared to males. Likewise, PERI and PRE groups exhibited areas of higher and lower CBF compared to males, chiefly lower CBF in ACC, medial and superior temporal gyrus, and basal ganglia, and higher CBF in parietal lobe.

ATP production. We investigated ATP/PCr using two separate mGLMs: model 1 included regions with higher $\mathrm{CBF}$ in POST versus $\mathrm{MALE}_{\mathrm{POST}}$ (ACC, insula and temporo-parietal regions); model 2 included regions with lower CMRglc and CBF in POST versus MALE $_{\text {POsT }}$ (frontal areas). Results are presented in Table 3 . With and without adjustment by APOE-4 status, the POST group had higher ATP/PCr than MALE POST $_{\text {in model } 1}$ $(p \leq 0.003)$ and borderline higher ATP/PCr in model $2(p \leq 0.082)$, and the PERI group had higher ATP/PCr than MALE $_{\text {PERI }}$ in model $2(p \leq 0.049)$. There were no differences between PRE and MALE $E_{\text {PRE }}$.

Amyloid- $\beta$ load. POST and PERI groups exhibited higher PiB uptake in AD-mask than MALE $\mathrm{POST}_{\text {and }}$

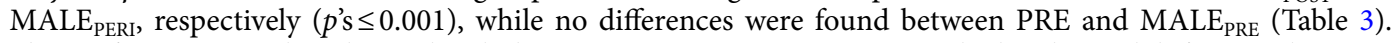
These effects remained unchanged including APOE-4 status as a covariate, which enhanced differences between PERI and MALE $E_{\text {PERI }}$ APOE- 4 carriers $(p=0.020)$. PiB differences between PERI and MALE $E_{\text {PERI }}$ were more pronounced among APOE-4 carriers (Table 3$)$.

Biomarker changes post-menopause. To examine whether biomarker effects were persistent postmenopause, and to test their specificity for menopause endocrine aging versus chronological aging, we performed 2-year follow-up volumetric MRI and FDG-PET scans in 17 POST and 12 MALE $_{\text {PosT }}$ (Supplementary Table S9). We first tested for changes in biomarkers within the brain regions showing POST-specific effects, and secondly, in the entire search volume.

Examination of brain regions exhibiting POST-stage effects on GMV indicated no GMV changes in inferior temporal and fusiform gyri, and GMV increases in the precuneus of the POST group (Fig. 4a, d; and Supplemen-

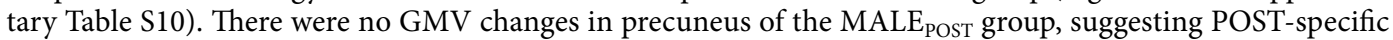

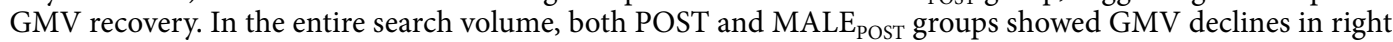
frontal gyrus, which did not differ between the groups (Supplementary Table S10).

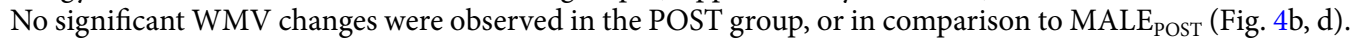


a
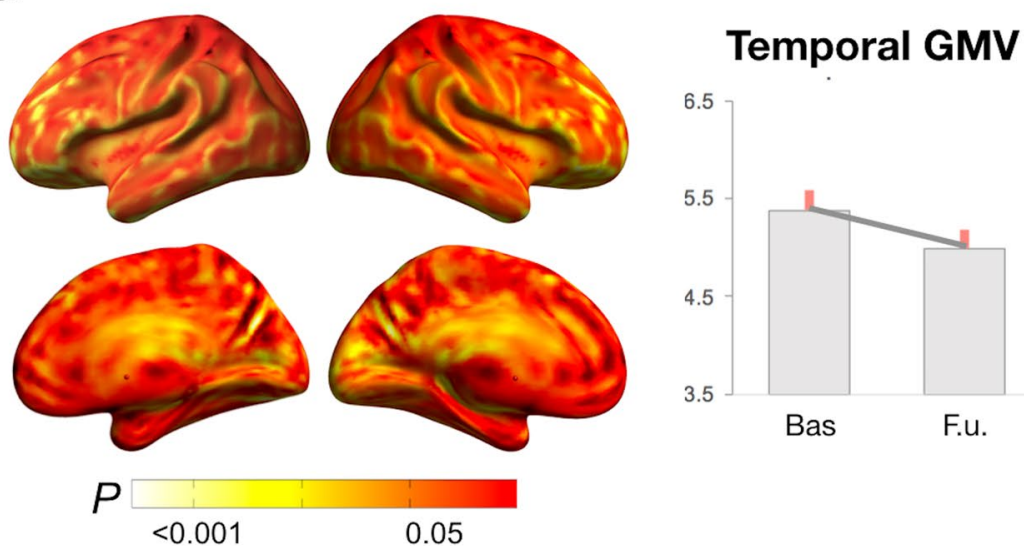

Precuneus GMV

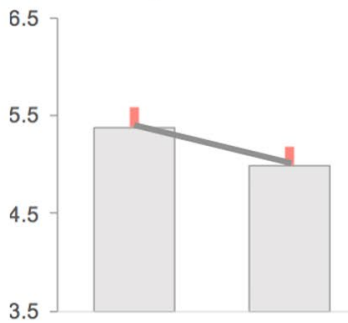

Bas

F.u.

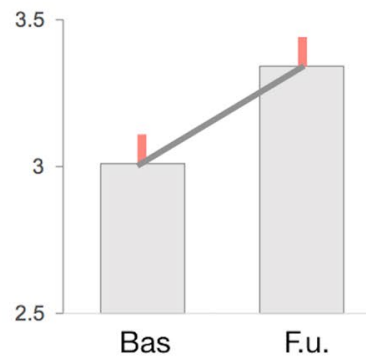

b
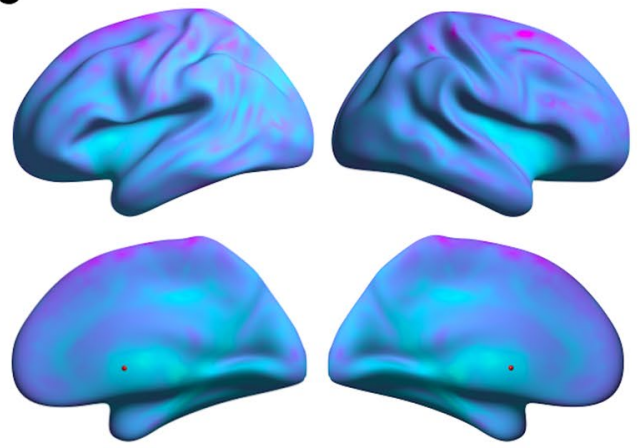

$P<0.001$

0.05
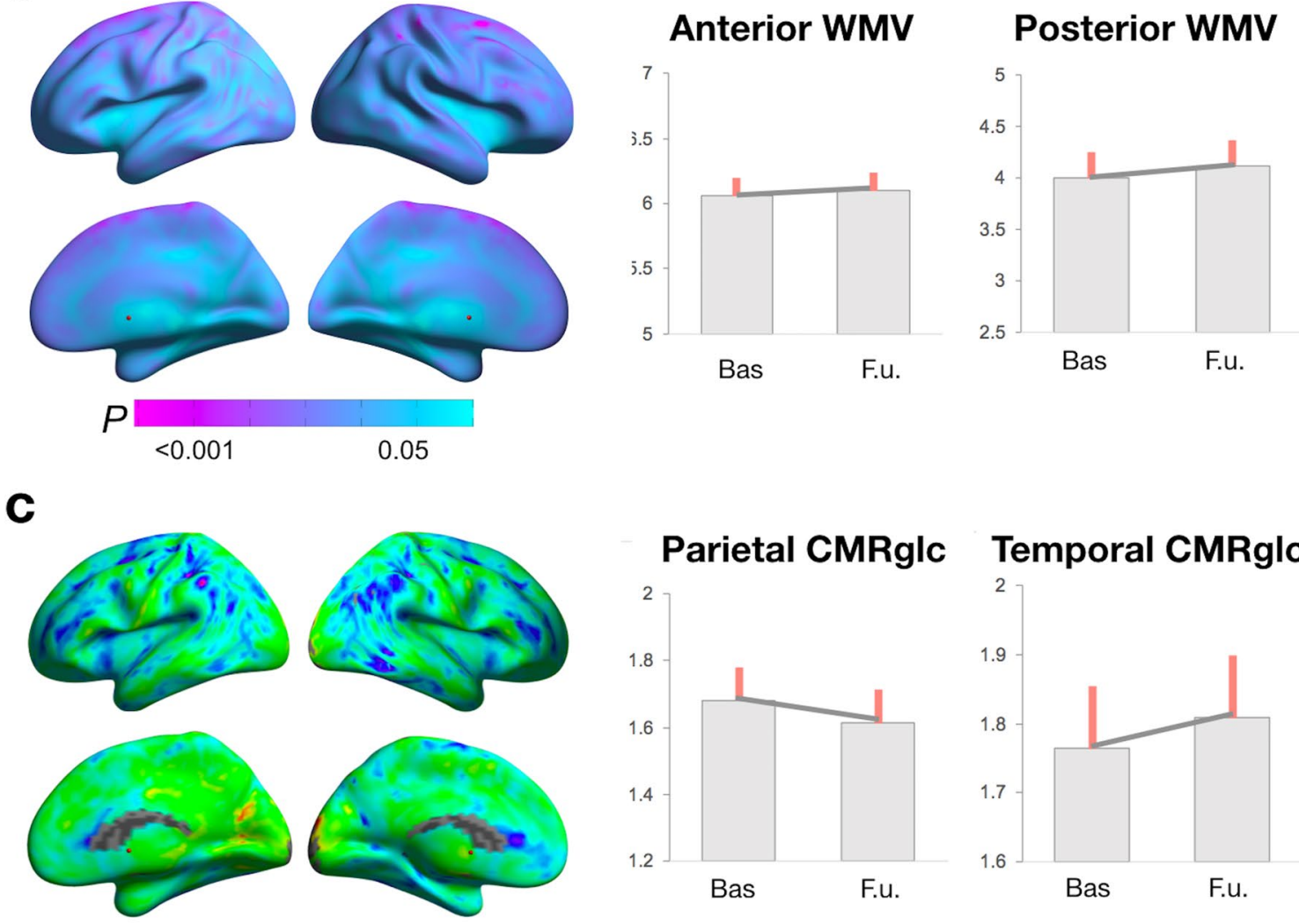

$P$

$<0.001$

0.05

Figure 4. Longitudinal biomarker changes post-menopause. (a) Surface maps of GMV change. (b) Surface maps of WMV change. (c) Surface maps of CMRglc change. (d) Plots representing mean (SE) biomarker levels at baseline (POST) versus 2-year follow-up (POST +2$)$ in the subset of POST participants with longitudinal brain scans. Surface maps are represented on modality-specific color-coded scales with corresponding $p$ values. See legends to Figs. 1 and 2.

Examination of temporo-parietal regions that were hypometabolic in the POST group versus PRE and PERI groups showed CMRglc declines in left inferior parietal lobule and not in other areas of the POST group (Fig. 4c, d; Supplementary Table S11). Parietal CMRglc declines were significant compared to MALE $E_{\text {POST }}$. In the entire search volume, there were no further longitudinal group differences.

Summary of biomarker findings. The main biomarker findings of this study are summarized in Fig. 5.

Sensitivity analysis. We performed a sensitivity analysis to test for effects of HT use and hysterectomy status on biomarker data. Results are presented in Supplementary Appendix. Briefly, including HT use and 


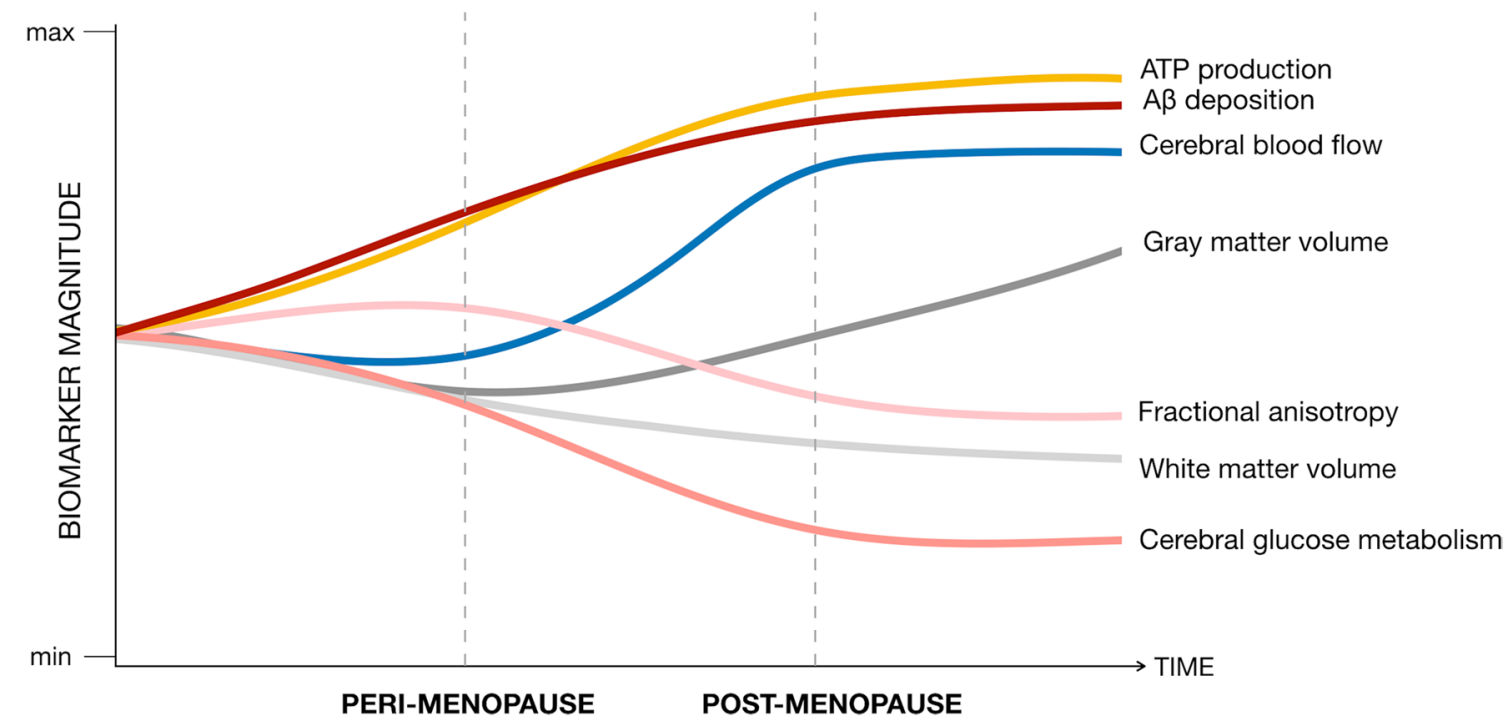

Figure 5. Summary of brain biomarker effects during the menopause transition. This figure summarizes the main results of the study by mapping estimated brain biomarker outcomes from pre-menopausal to perimenopausal and post-menopausal stages. Biomarker measures extracted from representative clusters for each modality are displayed on a standardized scale and normalized to pre-menopausal levels to enable examination of the magnitude of biomarker effects by menopausal stage and across modalities.

hysterectomy status as covariates left MT effects on brain biomarkers substantially unchanged. As compared to HT users, non-users exhibited lower GMV in inferior temporal gyrus, and lower CMRglc in parietal regions, independent of hysterectomy status. Restricting analysis to HT non-users confirmed results from the entire cohort, with two exceptions: CBF differences between MT groups were more pronounced among non-users, and FA differences between PERI and POST groups did not reach significance.

Associations between biomarkers and cognition. There were no significant differences in cognitive scores between MT groups (Table 1), or between each MT group and the corresponding age-matched male group (Supplementary Table S12). As shown in Supplementary Table S13, in the POST group, precuneus GMV was positively associated with memory scores at cross-section $(\mathrm{r}=0.311, p=0.007)$ and longitudinally $(\mathrm{r}=0.521$, $p=0.032)$. ATP/PCr in temporo-parietal regions was positively associated with global cognition $(\mathrm{Rho}=0.323$, $p=0.027)$. There were no significant associations between cognitive scores and regional biomarkers among PRE and PERI groups (Supplementary Table S13).

\section{Discussion}

Outcomes of this multi-modality neuroimaging analyses demonstrate that MT stage has pronounced effects on human brain's structure, connectivity, and energy metabolism, and provide a neurological framework for both vulnerability and resilience. Effects were most pronounced in regions subserving higher-order cognitive processes, and were independent of age, APOE-4 status, HT usage, and hysterectomy status. Comparisons to age-matched males provided further evidence that neuroimaging biomarker effects were specific to menopausal endocrine aging rather than to chronological aging. In most brain regions and across modalities, brain biomarkers stabilized or recovered post-menopause. Cognitive preservation post-menopause correlated with GMV recovery and brain ATP production, highlighting possible compensatory mechanisms. In parallel to the adaptive process, $A \beta$ deposition was more pronounced in PERI and POST women positive for APOE- 4 genotype, indicating specificity for $\mathrm{AD}$ risk with onset in the peri-menopause.

The MT is a normal physiological event. However, while the majority of women undergo menopause without long-term adverse effects ${ }^{1}$, many are vulnerable to the neurological shifts that can occur during this transition, experiencing bothersome symptoms ${ }^{2}$ as well as a higher risk of depression, anxiety, and $\mathrm{AD}^{13}$. Preclinical work indicates that the brain has the ability to compensate for changes in estrogen levels and estrogen receptor activity during the $\mathrm{MT}^{1}$. In some instances, however, compensatory responses are diminished, lacking, or limited to some estrogen-regulated networks ${ }^{1}$, which may account for the complex MT phenotype observed in women ${ }^{2}$. Investigation of compensatory responses is still in the early stages ${ }^{1}$, though clinical observations suggest that gradual hormonal changes during spontaneous MT may allow "brain resetting", e.g. neuronal adaptations to the hypo-estrogenic post-menopausal state ${ }^{1,14,15}$. Brain adaptation may account for the easing of menopausal symptoms like hot flashes, which tend to resolve 2-7 years into menopause ${ }^{2}$. Present neuroimaging results provide novel neurophysiological evidence for post-menopausal brain adaptation in humans, encompassing brain structure, connectivity and bioenergetics, and preservation of cognitive function.

Our results have several implications. First, compared to age-matched males, POST and PERI groups exhibited lower GMV in several cortical regions and subcortical structures such as hippocampus, amygdala, and 
thalamus. However, GMV generally stabilized post-menopause and selectively recovered in precuneus, an associative cortical area involved in social processes, episodic memory, and information integration ${ }^{16}$. On average, in the POST group, GMV in precuneus (a) was higher than the PERI group, (b) was comparable to age-matched males, and (c) increased over a 2-year span, a change that was not observed in males, indicating POST-specific recovery. Further, among POST participants, GMV in precuneus correlated with memory scores at cross-section and longitudinally, indicating that this area undergoes structural changes that are influenced by MT and have cognitive implications. Brain imaging studies of pregnancy, the other female-specific neuro-endocrine transition, also report dynamic GMV changes in precuneus, which are thought to reflect reduced neurogenesis in late pregnancy, followed by restoration by the time of weaning ${ }^{17}$. Although neurogenesis decreases with aging ${ }^{18}$, similar mechanisms may play a role in the brain's adaptation to the post-menopausal stage. Notably, the observed MT-related anatomical pattern maps onto estrogen-regulated systems ${ }^{1}$ and displays notable similarities to the neural networks impacted by pregnancy ${ }^{19}$.

MT stage also influenced WMV within major tracts connecting extensive parts of the cerebral cortex and subcortical regions. Both POST and PERI groups exhibited widespread WMV loss compared to age-matched males, with the POST group also exhibiting lower WMV in corona radiata compared to PRE and PERI groups. In spite of the WMV loss, all MT groups exhibited higher FA than males in corona radiata, as well as in fornix of the POST group, and in fornix, uncinate fasciculus and external capsule of the PERI group. PERI and PRE groups also exhibited some clusters with lower FA than males, involving chiefly SLF, whereas the POST group showed no deficits. To our knowledge, there are no previous DTI studies of the MT. However, there is evidence for sex differences in WM microstructure that vary with age and endocrine aging status. Most DTI studies on the entire age range found that males have generally higher FA than females in WM tracts such as SLF ${ }^{20}$, as in our comparisons with PERI and PRE groups. In contrast, during puberty (the first neuro-endocrine transition for both genders), females display higher FA than males in several WM tracts including corona radiata and fornix ${ }^{21}$. Animal studies report that pregnancy is also accompanied by increased regional water diffusivity ${ }^{22}$. While FA has a number of determinants, the principle factors are myelination and tissue architecture, with the inference that higher FA represents more "efficient" WM organization ${ }^{12}$. As such, our findings suggest greater efficiency of a smaller corona radiata and fornix post-menopause, raising the possibility that MT is accompanied by further refinement of these regions' connectivity. Longitudinal studies are warranted to map FA changes during the MT and in relation to other biomarkers.

The MT also impacted brain energetics on multiple levels. The POST group, and to a lesser extent the PERI group, exhibited hypometabolism in parieto-temporal cortices, consistent with previous reports in women at risk for $\mathrm{AD}^{9-11}$. However, in the present study, regional CMRglc largely plateaued post-menopause, suggesting adaptation to a new metabolic baseline after prolonged estrogen deficiency. Additionally, CBF and ATP production in temporo-parietal regions were elevated post-menopause, and ATP levels positively correlated with global cognition. Our findings of higher CBF in women than in men are consistent with the literature ${ }^{23}$, and further indicate that MT modulates CBF sex differences in midlife. To our knowledge, there are no previous studies of MT effects on brain ATP production in humans. Altogether, present results provide novel evidence for in vivo neurovascular-neurometabolic dissociations during the MT. Typically, regional brain activity, CMRglc, and $\mathrm{CBF}$ are coupled ${ }^{24}$. Dissociation can occur with aging, pathology, and inflammation ${ }^{24}$, or as a compensatory response $^{25}$. While data in humans are scarce, preclinical evidence indicates that estrogen loss during MT triggers CMRglc declines ${ }^{26}$, prompting an adaptive reaction to increase ketone bodies utilization as an alternative fuel for ATP $^{27}$. Continued reliance on ketones leads to compromised mitochondrial function, WM catabolism, and cellular apoptosis in animals ${ }^{26,27}$. In light of preclinical work, the higher CBF and ATP levels observed in our POST women may reflect a compensatory reaction to glucose hypometabolism, as well as a means to increase ketone metabolism.

We previously reported lower peripheral mitochondrial cytochrome oxidase activity (COX) in POST versus $\mathrm{PRE}$ women at risk for $\mathrm{AD}^{28}$. Herein, we investigated mitochondria ATP production in brain, which was higher in POST women independent of APOE-4 status, suggesting different mitochondrial activity profiles in brain and periphery. Animal models of menopause show dynamic relationships between central and peripheral metabolic systems, which tend to shift from uncoupled to coupled under metabolic stress ${ }^{29}$. Although brain mitochondria might eventually falter in older POST women, the positive associations between ATP production and global cognition suggest a recovery mechanism, at least in midlife. As aging and menopause differ to some extent between humans and animals, the neuro-energetics of menopause are also likely to differ - in this case, by supporting women's ability to transition into late life with preserved cognition, as discussed below.

In parallel to the hypothesized adaptive process, POST and PERI groups, especially APOE-4 carriers, exhibited higher $A \beta$ deposition compared to the PRE group and to age-matched males. While $A \beta$ deposition was mild, this data is consistent with previous studies of women at risk for $\mathrm{AD}^{9-11}$, and support evidence that interactions between age, female gender and APOE- 4 increase $\mathrm{AD}$ vulnerability during peri-menopause ${ }^{30}$. Chronologically, MT maps onto the preclinical phase of $\mathrm{AD}$, which begins decades prior to symptom onset ${ }^{31}$. The earlier onset of, and longer exposure to $\mathrm{A} \beta$ pathology may help explain the higher prevalence of $\mathrm{AD}$ in females, with POST women comprising over $60 \%$ of all cases ${ }^{32}$. It warrants emphasis that reproductive aging is not uniformly associated with $\mathrm{AD}$ risk. While some women in our cohort might eventually develop $\mathrm{AD}$, for others, $\mathrm{A} \beta$ deposition could reflect accelerated biological aging due to hormonal declines instead ${ }^{33}$. In fact, over $20 \%$ of healthy elderly display moderate cerebral $\mathrm{A} \beta$ burden and no dementia ${ }^{31}$.

The proportion of APOE-4 carriers in our study was $42 \%$, which is higher than the $15-30 \%$ observed in the general population ${ }^{34}$. Many of our participants volunteer because of concerns about cognitive functioning, which tend to be more frequent among APOE- 4 carriers $^{35}$. As such, our cohort may be self-enriched with individuals at higher a priori risk of AD. Although our results were independent of APOE-4 status, more studies are needed to replicate these findings in community-dwelling individuals randomly recruited from the population. 
The observed MT effects were independent of HT and hysterectomy status, though HT use was associated with mild beneficial effects on GMV and CMRglc, consistent with some clinical trials ${ }^{6}$. Whether HT provides protection against cognitive aging and $\mathrm{AD}$ remains unclear. Observational studies generally found positive effects on cognition across multiple HT regimens ${ }^{36}$, whereas clinical trials of late POST women aged 65 or older reported an increased dementia risk with estrogen-plus-progestin HT, and no effects with estrogen-alone ${ }^{37}$, while studies of early POST women observed no adverse or beneficial effects on cognition ${ }^{38,39}$. Overall, HT's efficacy is thought to depend on timing of treatment initiation with respect to age at menopause, with benefits pertaining to early initiation, especially after induced menopause ${ }^{7,37}$. Our neuroimaging results point to the MT as a dynamic neurological process and, therefore, a window of vulnerabilities and opportunities when the human brain is influenced by biochemical adjustments, but is also likely to be receptive to interventions. More studies are needed to test the efficacy of HT instituted prior to menopause, and in relation to brain biomarker levels.

In our study, POST participants did not exhibit impaired cognitive performance as compared to the other groups. While self-reports of poor memory and concentration are common in women undergoing $\mathrm{MT}^{2}$, menopause itself hasn't been associated with clinically significant functional impairment or deficits on cognitive testing ${ }^{40}$. Further, it is well-documented that women perform better than men on several cognitive domains across the adult lifespan- an advantage that seems to persist even after a dementia diagnosis ${ }^{41}$. Lack of cognitive decline may seem in contrast with neuroimaging findings of extensive MT-stage effects on brain biomarkers. In the present study, GMV recovery and ATP production positively correlated with cognitive scores in the POST group. While this awaits confirmation, present results provide neurophysiological insights on brain adaptation during the MT, which may at least in part, account for the lack of cognitive impairment among POST women ${ }^{40}$. Large-scale epidemiological studies reported a decrement in cognitive performance during the peri-menopause, followed by a rebound to pre-menopausal levels in post-menopause ${ }^{42}$. This is consistent with the post-menopausal brain biomarker recovery observed in our study. Additionally, studies that differentiated between early and late menopausal stages reported subtle yet transitory cognitive changes during the $\mathrm{MT}^{43}$, which have yet to be investigated with neuroimaging. However, it is possible that brain and cognitive aging trajectories may differ among MT women depending on genetic predisposition, medical history, and environmental influences. More longitudinal imaging studies with larger samples are warranted to address these questions. Additionally, as our cohort was highly educated, the observed lack of cognitive deficits may not be generalizable to women with different educational or socio-economical background.

Another limitation of this study is the low percentage of minority participants, which limits the generalizability of our findings. Today, no brain imaging studies have been conducted to investigate MT effects on brain biomarkers based on ethnicity. Multiple studies have indicated increased frequency and severity of menopausal symptoms, particularly vasomotor symptoms, with greater prevalence in African-American and Hispanic women $^{44}$, which strongly argues for studies that specifically address differences in MT-associated biomarker outcomes across ethnic groups.

From a methodological perspective, we examined statistically powered groups of women at different MT stages, paired with age correction procedures including comparisons to age-matched males and longitudinal evaluations in a subset of participants. Nonetheless, a causal link between MT and brain biomarkers cannot be unequivocally established. We chose this study design because the timing of menopause is highly variable, with a median age at menopause of 51 years, and a distribution of 40-58 years ${ }^{2}$. Longitudinal studies may require $>10$ years of follow-ups to capture the effects of MT on brain biomarkers. While studies of surgical menopause ideally reduce follow-up times, the procedure seems associated with different, possibly more severe outcomes $^{45,46}$. Longitudinal studies are warranted to replicate our findings and test for differential effects of induced and spontaneous menopause.

Determination of MT status was based on established diagnostic criteria ${ }^{47}$. Nonetheless, some PERI may have been early POST, while some early POST could have been late PERI. This would, however, conservatively reduce power in detecting $\mathrm{MT}$-stage effects. Considering the hormonal changes women experience during MT, and the observed biomarker effects, we attribute our results to the endocrine climate of menopause. Our analyses were corroborated by testing of males of similar demographic and socio-economical characteristics. Future studies tracking changes in hormones, medical status, and lifestyle are needed to clarify the complex relationships between MT and brain aging ${ }^{13}$, and identify which factors impact successful versus unsuccessful brain adaptation post-menopause.

Overall, present findings show that human menopause is a dynamic neurological transition that reshapes the neural landscape of the female brain during midlife endocrine aging, and provide preliminary evidence for an adaptive process serving the transition into late life.

\section{Methods}

Participants. This is a non-randomized natural history non-treatment study of healthy, cognitively normal female and male participants ages 40-65 years at different menopausal stages, recruited at Weill Cornell Medicine (WMC) and NYU School of Medicine between 2015 and 2020. Participants were recruited by self-referral, flyers, and word of mouth, as described ${ }^{9-11}$. Pre-established exclusion criteria included medical conditions that may affect brain structure or function (e.g. stroke, any neurodegenerative diseases, major psychiatric disorders, hydrocephalus, white matter lesions suggestive of demyelinating disease such as Multiple Sclerosis, intracranial mass and infarcts on MRI), use of psychoactive medications, and contraindications to MRI or PET. Participants had Mini Mental Status Exam $\geq 27$ and normal cognitive performance for age and education ${ }^{9-11}$.

The patients' sex was determined by self-report. APOE genotype was assessed using quantitative Polymerase Chain Reaction (qPCR) procedures ${ }^{9-11}$. Participants carrying one or two copies of APOE-4 allele were grouped as carriers, and compared to non-carriers. 
Standard protocol approvals, registrations, and patient consents. All methods were carried out in accordance with relevant guidelines and regulations. All experimental protocols were approved by the WMC and NYU Institutional Review Boards. Written informed consent was obtained from all participants.

Cognitive testing. Our neuropsychological testing battery included tests measuring three cognitive domains: memory (immediate and delayed recall of a paragraph and paired associates), higher-order processing (block design tests), and language (object naming) ${ }^{9-11}$. We computed (i) a global cognitive score by creating Z-scores within each domain and averaging across the domains; and (ii) a memory score by creating Z-scores for each memory test and averaging across tests.

Menopause assessments. Determination of menopausal status was based on the Stages of Reproductive Aging Workshop (STRAW) criteria $^{47}$ and corroborated by means of hormone assessments. Female participants were classified as regular cyclers (PRE), irregular cyclers (PERI), and no cycle for 12 or more months (POST).

Brain imaging acquisition and analysis. All participants underwent structural MRI, and 160 participants received ${ }^{18} \mathrm{~F}-\mathrm{FDG}$ and ${ }^{11} \mathrm{C}-\mathrm{PiB}$ PET at WMC. In 2017, we started acquiring DTI, ASL, and MRS scans, which have been done on 100 participants.

Our protocol included three MRI sequences on a 3.0 T G.E. Discovery MR750 scanner: (a) sagittal T1-MRI [Brain Volume Imaging (BRAVO); $1.0 \times 1.0 \times 1.0 \mathrm{~mm}$ resolution, $8.2 \mathrm{~ms}$ repetition time (TR), $3.2 \mathrm{~ms}$ echo time (TE), $25.6 \mathrm{~cm}$ field of view (FOV), $256 \times 256$ matrix] used to estimate GMV and WMV; (b) DTI scan [b=0 s/ $\mathrm{mm}^{2}, \mathrm{~b}=1000 \mathrm{~s} / \mathrm{mm} 255$-directions, $8000 \mathrm{~ms}$ TR, $65 \mathrm{~ms}$ TE, $256 \times 256$ matrix, $0.9 \times 0.9 \times 1.8 \mathrm{~mm}$ resolution]; (c) ASL scan [pseudo-continuous technique with $4851 \mathrm{~ms}$ TR, $10.6 \mathrm{~ms}$ TE, 4 averages, $24 \mathrm{~cm}$ FOV, $2.0 \times 2.0 \times 3.8 \mathrm{~mm}$ resolution] used to estimate $\mathrm{CBF}$ using arterial blood water ${ }^{48}$.

${ }^{31} \mathrm{P}$-MRS was acquired on the GE scanner using a dual tuned 32 -channel ${ }^{31} \mathrm{P} /{ }^{1} \mathrm{H}$ quadrature head coil $[2048$ points, $5000 \mathrm{~Hz}$ sweep width, $2000 \mathrm{~ms}$ TR, 2 averages, $55^{\circ}$ flip angle at $51.3 \mathrm{MHz}, 24 \mathrm{~cm} \mathrm{FOV]} \mathrm{to} \mathrm{assess} \mathrm{mito-}$ chondrial function in brain through the mapping of intracellular ATP and PCr levels ${ }^{49,50}$. A higher ATP/PCr ratio reflects greater ATP production relative to utilization ${ }^{51}$. Shimming was performed using a ${ }^{1} \mathrm{H}$ single voxel technique placed over the entire brain. Raw data was processed using Hamming and Fermi k-space filters, $20 \mathrm{~Hz}$ exponential filtering and zero-filling in time, $\mathrm{x}$ and $\mathrm{y}$-domains prior to 3D Fast Fourier Transformation. The PCr peak is set at $0.0 \mathrm{ppm}$ and susceptibility corrections performed. Baseline correction was applied by an experienced analyst (JPD). This resulted in a $16 \times 16$ image of $1.5 \times 1.5 \times 3.0 \mathrm{~cm}$ voxels with the signal intensity in each voxel corresponding to the peak area of the ${ }^{31} \mathrm{P}$ metabolite. The central 4 slices of Chemical Shift Imaging (CSI) data were then registered to the BRAVO sequence.

PET scans were acquired on a Siemens BioGraph mCT 64-slice PET/CT operating in 3D mode [70 cm transverse FOV, $16.2 \mathrm{~cm}$ axial FOV] following standardized procedures ${ }^{9-11}$. Summed images were obtained 40-60 min post-injection of $5 \mathrm{mCi}$ of ${ }^{18} \mathrm{~F}-\mathrm{FDG}$, and $60-90 \mathrm{~min}$ post-injection of $15 \mathrm{mCi}$ of ${ }^{11} \mathrm{C}-\mathrm{PiB}$. All images were corrected for attenuation, scatter and decay, and smoothed for uniform resolution ${ }^{52}$.

Two-year follow-up volumetric MRI and FDG-PET scans were performed on 17 POST women (age at baseline scan: $57 \pm 3$ years; time to follow-up: $2.2 \pm 0.4$ years) and 12 males within the same age range (age at baseline: $56 \pm 4$ years, time to follow-up: $2.4 \pm 0.4$ years) using the same protocol and equipment as the baseline exams.

Multiparametric mapping. All images were processed in Statistical Parametric Mapping (SPM12, (https://www. fil.ion.ucl.ac.uk/spm/software/spm 12/) and Matlab 7.8, using a fully automated image processing pipeline ${ }^{9-11}$. For each participant, scans were co-registered to the T1-MRI and to each other using the Normalized Mutual Information routine ${ }^{53}$. Volumetric scans were processed with voxel-based morphometry (VBM), including Jacobian modulation to restore volumes using the unified segmentation algorithm, DARTEL normalization of the segments, and application of an $8 \mathrm{~mm}$ full-width at half maximum (FWHM) smoothing kernel ${ }^{53}$. Co-registered DTI, ASL, and PET scans were spatially normalized using subject-specific transformation matrices obtained from the corresponding MRI and smoothed at 10-mm FWHM. For longitudinal analysis, each participant's MRIs were processed using longitudinal routines incorporating rigid-body registration, intensity inhomogeneity correction, and nonlinear diffeomorphic registration ${ }^{53}$. Co-registered follow-up PET were processed using the baseline MRI as the anchor.

ATP/PCr and PiB uptake were quantified using FreeSurfer 6.0 and Desikan-Killiany Atlas-based cortical ROIs $^{54,55}$ applied to the aligned MRI. For PiB analysis, we created an AD-mask by averaging parietal, temporal, frontal, posterior cingulate and precuneus $\mathrm{ROIs}^{56,57}$. PiB uptake in $\mathrm{AD}$-mask was normalized to cerebellar GM uptake obtained via FreeSurfer.

Statistical analysis. Analyses were performed in SPSS v.25 and SPM12. Clinical, demographic and cognitive measures were examined with General Linear Models (GLM) or Chi-squared tests at $p<0.05$.

To address the age difference between POST and PRE groups we ${ }^{11,58}$ : (a) used box plots and frequency diagrams to ensure that we had sufficient age overlap among women of different MT statuses, which enabled us to examine the effects of endocrine aging separately from those of chronological aging; (b) included age as a covariate in all analyses; (c) compared each MT group to an age-range matched male group (MALE $\mathrm{PRE}_{\text {, MALE }}$ PERI, MALE $_{\mathrm{POST}}$ ); and (d) compared 2-year MRI and FDG-PET changes in a subset of POST women and MALE $\mathrm{POST}_{\text {. }}$

All images were analyzed using SPM12, except for MRS and PiB-PET ROI data which were examined using SPSS, as described below. SPM12 analyses were adjusted by age, APOE- 4 status, and modality-specific confounds, e.g. GMV, WMV and FA were adjusted by total intracranial volume (TIV) obtained via Freesurfer, CMRglc by global metabolic activity, CBF by global CBF. Statistical maps were conservatively obtained by first applying an a 
priori masking image including regions involved in the brain estrogen network with known cognitive functions ${ }^{1}$, and then a stringent cluster-level FWE correction at $p<0.05$, with cluster extent $\geq 20$ voxels. The masking image comprised anterior and posterior cingulate cortex; fusiform gyrus; inferior and medial orbitofrontal cortex; inferior, medial, middle and superior frontal cortex; inferior, middle, and superior temporal gyrus; inferior and superior parietal lobule; insula; medial temporal lobe; precuneus; putamen; and thalamus. For WM analysis, the brainstem was also included. Further, results were examined after application of an a priori GM or WM mask to restrict analysis to GM or WM fiber voxels, respectively. Identification of GM clusters was made using MNI coordinates, and of WM clusters by reference to the Johns Hopkins University White-Matter Labels atlas ${ }^{59}$.

In figures, statistical parametric maps and surface maps were obtained using SPM12 and SurfRend v.1 (http:// spmsurfrend.sourceforge.net/).

Biomarker differences between MT groups. For SPM12 analyses, we used full factorial models to test for biomarker differences between MT groups. If a significant main effect was found, we then proceeded to separately examine the directionality of biomarker differences between paired groups using post-hoc $t$-contrasts. For all biomarker modalities, statistical parametric maps of significant results were saved as masking images, which were then used as implicit masks of MT stage-specific regional biomarker effects in subsequent analyses (see b and c, below). For example, the POST group showed larger GMV in precuneus compared to PERI. The precuneus cluster was saved as a masking image for further examination of POST and PERI-specific GMV effects in this region.

For ROI analyses, we used (i) mGLMs with post-hoc Sidak tests to test for group differences in ATP/PCr in regions showing MT effects on CMRglc and CBF (e.g. temporo-parietal regions), adjusting by age and APOE-4 status as covariates, at $p<0.05$; and (ii) GLMs with post-hoc Sidak tests to examine AD-mask PiB uptake for MT group effects, and for APOE-4 effects by (i) examining APOE- 4 status as a covariate; (ii) testing for interactions between MT and APOE-4 status; and (iii) for MT effects within each APOE-4 subgroup, at $p<0.05$.

Comparisons to age-matched males. For SPM12 analyses, we used two-sample t-tests to compare each MT group to the corresponding age-matched male group, using the same confounders as above. For all biomarker modalities, our main endpoint was detection of sex-related biomarker differences within the implicit masks of MT stage-specific effects described in (a). For example, we used the precuneus cluster described above to test for GMV differences between POST and PERI groups and corresponding age-matched male groups. Comparison to MALE $_{\text {PERI }}$ confirmed presence of lower precuneus GMV in the PERI group, while comparison to MALE $E_{\text {POST }}$ showed no significant differences in precuneus of the POST group, suggesting POST-specific GMV recovery. As this analysis was restricted to specific clusters, our secondary endpoint was detection of group differences in the entire search volume.

For ROI analyses, we used (i) mGLMs with post-hoc Sidak tests to test for ATP/PCr group differences in regions showing CMRglc and CBF differences between POST and MALE POST; $_{\text {; }}$ and (ii) GLMs with post-hoc Sidak to examine $\mathrm{AD}$-mask $\mathrm{PiB}$ uptake for group effects and for APOE-4 status effects (as described above), at $p<0.05$.

Biomarker changes post-menopause. We investigated whether the structural and CMRglc effects of menopause

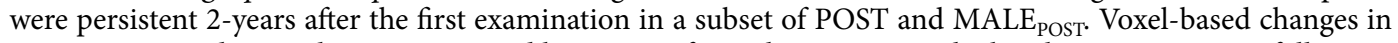
GMV, WMV and CMRglc were examined by means of post-hoc $t$-tests on the baseline versus 2-year follow-up scans for each group, and by comparing the maps of change between groups, using SPM12. This analysis allowed us to identify brain regions showing (i) significant biomarker changes over time in each group, and (ii) different rates of change in POST versus MALE POST $_{\text {. }}$

Our main endpoint was detection of biomarker changes within the implicit masks of POST-specific regional effects, as described in (a). Therefore, we first tested for longitudinal changes within those pre-specified regions of the POST group, and then compared the rate of change in those regions between POST and MALE $\mathrm{POST}_{\text {. For }}$ example, the POST group showed larger precuneus GMV compared to PERI. Longitudinally, the POST group

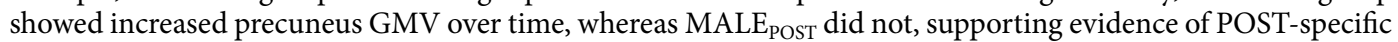
GMV recovery in this region. We then tested for longitudinal differences in the entire search volume, e.g. biomarker changes outside of the implicit masks. Results were examined at $p<0.05$, cluster-level corrected, adjusting for time to follow-up and modality-specific confounds.

Cognitive measures: group effects and biomarker associations. We examined cognitive measures for differences across groups using Kruskal-Wallis non-parametric tests for global cognition as this measure did not follow a normal distribution, and GLMs with post-hoc Sidak tests for analysis of memory scores, adjusting by age, education, and APOE- 4 status, at $p<0.05$. Cognitive measures were then tested for correlations with biomarker data. Since cognitive measures were preserved in the POST group, we tested for associations between cognitive measures and regional biomarkers showing preservation in the POST group, e.g. precuneus GMV, temporo-parietal ATP/PCr and CBF. For descriptive purposes, we examined these associations also among PRE and PERI groups. Spearman's Rho tests were used to test for correlations with global cognition, and Pearson's $r$ tests for correlations with memory scores, at $p<0.05,2$-tailed.

\section{Data availability}

The datasets analyzed during the current study may be made available from the corresponding author on reasonable request. 
Received: 12 January 2021; Accepted: 6 May 2021

Published online: 09 June 2021

\section{References}

1. Brinton, R. D., Yao, J., Yin, F., Mack, W. J. \& Cadenas, E. Perimenopause as a neurological transition state. Nat Rev Endocrinol 11, 393-405. https://doi.org/10.1038/nrendo.2015.82 (2015).

2. Monteleone, P., Mascagni, G., Giannini, A., Genazzani, A. R. \& Simoncini, T. Symptoms of menopause-global prevalence, physiology and implications. Nat Rev Endocrinol. 14, 199-215. https://doi.org/10.1038/nrendo.2017.180 (2018).

3. McEwen, B. S., Alves, S. E., Bulloch, K. \& Weiland, N. G. Ovarian steroids and the brain: implications for cognition and aging. Neurology 48, 8S-15S (1997).

4. Yue, X. et al. Brain estrogen deficiency accelerates Abeta plaque formation in an Alzheimer's disease animal model. Proc Natl Acad Sci U S A 102, 19198-19203. https://doi.org/10.1073/pnas.0505203102 (2005).

5. Yao, J. et al. Mitochondrial bioenergetic deficit precedes Alzheimer's pathology in female mouse model of Alzheimer's disease. Proc Natl Acad Sci U S A 106, 14670-14675. https://doi.org/10.1073/pnas.0903563106 (2009).

6. Comasco, E., Frokjaer, V. G. \& Sundström-Poromaa, I. Functional and molecular neuroimaging of menopause and hormone replacement therapy. Front Neurosci 8, 388. https://doi.org/10.3389/fnins.2014.00388 (2014).

7. Maki, P. M. The timing of estrogen therapy after ovariectomy-implications for neurocognitive function. Nat Clin Pract Endocrinol Metabol 4, 494 (2008).

8. Bertram, L. \& Tanzi, R. E. Thirty years of Alzheimer's disease genetics: the implications of systematic meta-analyses. Nat Rev Neurosci 9, 768-778. https://doi.org/10.1038/nrn2494 (2008).

9. Mosconi, L. et al. Increased Alzheimer's risk during the menopause transition: a 3-year longitudinal study. PloS One 13, e0207885 (2018).

10. Mosconi, L. et al. Sex differences in Alzheimer risk Brain imaging of endocrine vs chronologic aging. Neurology 89, 1382-1390 (2017).

11. Rahman, A. et al. Sex-driven modifiers of Alzheimer risk. Neurology 95, e166. https://doi.org/10.1212/WNL.0000000000009781 (2020).

12. Le Bihan, D. et al. Diffusion tensor imaging: concepts and applications. J Magn Reson Imaging 13, 534-546. https://doi.org/10. 1002/jmri.1076 (2001).

13. Rahman, A. et al. Sex and gender driven modifiers of Alzheimer's: the role for estrogenic control across age, race, medical, and lifestyle risks. Front Aging Neurosci https://doi.org/10.3389/fnagi.2019.00315 (2019).

14. Deecher, D. C. \& Dorries, K. Understanding the pathophysiology of vasomotor symptoms (hot flushes and night sweats) that occur in perimenopause, menopause, and postmenopause life stages. Arch Womens Ment Health 10, 247-257. https://doi.org/10.1007/ s00737-007-0209-5 (2007).

15. Rossmanith, W. G. \& Ruebberdt, W. What causes hot flushes? The neuroendocrine origin of vasomotor symptoms in the menopause. Gynecol Endocrinol 25, 303-314. https://doi.org/10.1080/09513590802632514 (2009).

16. Cavanna, A. E. \& Trimble, M. R. The precuneus: a review of its functional anatomy and behavioural correlates. Brain 129, 564-583. https://doi.org/10.1093/brain/awl004 (2006).

17. Hoekzema, E. et al. Pregnancy leads to long-lasting changes in human brain structure. Nat Neurosci. 20, 287-296. https://doi.org/ 10.1038/nn.4458 (2017).

18. Gross, C. G. Neurogenesis in the adult brain: death of a dogma. Nat Rev Neurosci. 1, 67-73. https://doi.org/10.1038/35036235 (2000).

19. Brunton, P. J. \& Russell, J. A. The expectant brain: adapting for motherhood. Nat Rev Neurosci 9, 11-25. https://doi.org/10.1038/ nrn2280 (2008).

20. Kanaan, R. A. et al. Gender differences in white matter microstructure. PLoS ONE 7, e38272. https://doi.org/10.1371/journal.pone. 0038272 (2012).

21. Bava, S. et al. Sex differences in adolescent white matter architecture. Brain Res 1375, 41-48. https://doi.org/10.1016/j.brainres. 2010.12.051 (2011).

22. Chan, R. W. et al. Structural and functional brain remodeling during pregnancy with diffusion tensor MRI and resting-state functional MRI. PLoS ONE 10, e0144328. https://doi.org/10.1371/journal.pone.0144328 (2015).

23. Aanerud, J. et al. Brain energy metabolism and blood flow differences in healthy aging. J Cerebr Blood Flow Metabol 32, 1177-1187 (2012).

24. Iadecola, C. Neurovascular regulation in the normal brain and in Alzheimer's disease. Nat Rev Neurosci 5, 347-360. https://doi. org/10.1038/nrn1387 (2004).

25. Masamoto, K. New horizons in neurovascular coupling: a bridge between brain circulation and neural plasticity, 1st edn Vol. 225 (Elsevier Science, Amsterdam, 2016).

26. Brinton, R. D. The healthy cell bias of estrogen action: mitochondrial bioenergetics and neurological implications. Trends Neurosci 31, 529-537. https://doi.org/10.1016/j.tins.2008.07.003 (2008).

27. Ding, F., Yao, J., Rettberg, J. R., Chen, S. \& Brinton, R. D. Early decline in glucose transport and metabolism precedes shift to ketogenic system in female aging and Alzheimer's mouse brain: implication for bioenergetic intervention. PLoS ONE 8, e79977. https://doi.org/10.1371/journal.pone.0079977 (2013).

28. Mosconi, L. et al. Perimenopause and emergence of an Alzheimer's bioenergetic phenotype in brain and periphery. PloS One 12, e0185926 (2017)

29. Wang, Y. et al. Midlife chronological and endocrinological transitions in brain metabolism: system biology basis for increased Alzheimer's risk in female brain. Sci Rep 10, 8528. https://doi.org/10.1038/s41598-020-65402-5 (2020).

30. Riedel, B. C., Thompson, P. M. \& Brinton, R. D. Age, APOE and sex: triad of risk of Alzheimer's disease. J Steroid Biochem Mol Biol 160, 134-147. https://doi.org/10.1016/j.jsbmb.2016.03.012 (2016).

31. Sperling, R. A., Karlawish, J. \& Johnson, K. A. Preclinical Alzheimer disease-the challenges ahead. Nat Rev Neurol. 9, 54-58. https:// doi.org/10.1038/nrneurol.2012.241 (2013).

32. Ferretti, M. T. et al. Sex differences in Alzheimer disease-the gateway to precision medicine. Nat Rev Neurol 14, 457-469 (2018).

33. Levine, M. E. et al. Menopause accelerates biological aging. Proc Natl Acad Sci 113, 9327-9332. https://doi.org/10.1073/pnas.16045 58113 (2016).

34. Singh, P. P., Singh, M. \& Mastana, S. S. APOE distribution in world populations with new data from India and the UK. Ann Hum Biol. 33, 279-308. https://doi.org/10.1080/03014460600594513 (2006).

35. Small, G. W. et al. Memory self-appraisal in middle-aged and older adults with the apolipoprotein E-4 allele. Am J Psychiatry 156, 1035-1038 (1999).

36. Morrison, J. H., Brinton, R. D., Schmidt, P. J. \& Gore, A. C. Estrogen, menopause, and the aging brain: how basic neuroscience can inform hormone therapy in women. J Neurosci. 26, 10332-10348. https://doi.org/10.1523/jneurosci.3369-06.2006 (2006).

37. Lobo, R. A. Hormone-replacement therapy: current thinking. Nat Rev Endocrinol. 13, 220-231. https://doi.org/10.1038/nrendo. 2016.164 (2017). 
38. Miller, V. M. et al. The Kronos early estrogen prevention study (KEEPS): what have we learned?. Menopause 26, 1071-1084. https:// doi.org/10.1097/gme.0000000000001326 (2019).

39. Henderson, V. W. et al. Cognitive effects of estradiol after menopause: a randomized trial of the timing hypothesis. Neurology 87, 699-708 (2016).

40. Maki, P. M. \& Henderson, V. W. Cognition and the menopause transition. Menopause 23, 803-805. https://doi.org/10.1097/gme. 0000000000000681 (2016).

41. Rentz, D. M. et al. Sex differences in episodic memory in early midlife: impact of reproductive aging. Menopause 24, 400-408 (2017).

42. Greendale, G. A. et al. Effects of the menopause transition and hormone use on cognitive performance in midlife women. Neurology 72, 1850-1857. https://doi.org/10.1212/WNL.0b013e3181a71193 (2009).

43. Weber, M. T., Rubin, L. H. \& Maki, P. M. Cognition in perimenopause: the effect of transition stage. Menopause 20, 511-517. https://doi.org/10.1097/GME.0b013e31827655e5 (2013).

44. Obermeyer, C. M. Menopause across cultures: a review of the evidence. Menopause 7, 184-192. https://doi.org/10.1097/00042 192-200007030-00009 (2000).

45. Rocca, W. A., Grossardt, B. R. \& Shuster, L. T. Oophorectomy, estrogen, and dementia: a 2014 update. Mol Cell Endocrinol. 389, 7-12 (2014).

46. Zeydan, B. et al. Association of bilateral salpingo-oophorectomy before menopause onset with medial temporal lobe neurodegeneration. JAMA Neurol. 76, 95-100. https://doi.org/10.1001/jamaneurol.2018.3057 (2019).

47. Harlow, S. D. et al. Executive summary of the stages of reproductive aging workshop + 10: addressing the unfinished agenda of staging reproductive aging. Menopause 19, 387-395. https://doi.org/10.1097/gme.0b013e31824d8f40 (2012).

48. Wang, J. et al. Arterial transit time imaging with flow encoding arterial spin tagging (FEAST). Magn Reson Med 50, 599-607. https://doi.org/10.1002/mrm.10559 (2003).

49. Rijpma, A., van der Graaf, M., Meulenbroek, O., Olde Rikkert, M. G. M. \& Heerschap, A. Altered brain high-energy phosphate metabolism in mild Alzheimer's disease: a 3-dimensional 31P MR spectroscopic imaging study. NeuroImage Clin 18, 254-261. https://doi.org/10.1016/j.nicl.2018.01.031 (2018).

50. Du, F. et al. Tightly coupled brain activity and cerebral ATP metabolic rate. Proc Natl Acad Sci. 105, 6409-6414. https://doi.org/ 10.1073/pnas.0710766105 (2008).

51. Pettegrew, J. W. et al. Correlation of phosphorus-31 magnetic resonance spectroscopy and morphologic findings in Alzheimer's disease. Arch Neurol 45, 1093 (1988).

52. Joshi, A., Koeppe, R. A. \& Fessler, J. A. Reducing between scanner differences in multi-center PET studies. Neuroimage 46, 154-159. https://doi.org/10.1016/j.neuroimage.2009.01.057 (2009).

53. Ashburner, J. \& Friston, K. J. Voxel-based morphometry-the methods. Neuroimage 11, 805-821. https://doi.org/10.1006/nimg. $2000.0582(2000)$.

54. Desikan, R. S. et al. An automated labeling system for subdividing the human cerebral cortex on MRI scans into gyral based regions of interest. Neuroimage 31, 968-980. https://doi.org/10.1016/j.neuroimage.2006.01.021 (2006).

55. Fischl, B. FreeSurfer. NeuroImage 62, 774-781. https://doi.org/10.1016/j.neuroimage.2012.01.021 (2012)

56. Vlassenko, A. G. et al. Imaging and cerebrospinal fluid biomarkers in early preclinical alzheimer disease. Ann Neurol. 80, 379-387. https://doi.org/10.1002/ana.24719 (2016).

57. Mintun, M. A. et al. [11C]PIB in a nondemented population: potential antecedent marker of Alzheimer disease. Neurology 67, 446-452. https://doi.org/10.1212/01.wnl.0000228230.26044.a4 (2006).

58. Becker, J. B. et al. Strategies and methods for research on sex differences in brain and behavior. Endocrinology 146, 1650-1673. https://doi.org/10.1210/en.2004-1142 (2005).

59. Hua, K. et al. Tract probability maps in stereotaxic spaces: analyses of white matter anatomy and tract-specific quantification. Neuroimage 39, 336-347. https://doi.org/10.1016/j.neuroimage.2007.07.053 (2008).

\section{Acknowledgements}

This study was supported by grants from NIH/NIA (P01AG026572, R01AG057931, R01AG035137, R01AG13616), NIH/NCATS UL1TR002384, the Cure Alzheimer's Fund, Maria Shriver's Women's Alzheimer's Movement; and the generous support of Harold W. McGraw III and Nancy G. McGraw, and Carol and Michael Weisman. In memory of Dr. Bruce S. McEwen.

\section{Author contributions}

L.M., R.B.D.: Conceived the idea and planned the research; J.D., E.S., S.J., L.L., G.J., A.R., H.H., S.P., R.I.: contributed to data collection; L.M., V.B., J.D., R.B.D.: conceptually contributed to the design of experiments; L.M., V.B., J.D., R.A., D.M.: participated in data analysis; and L.M., V.B., J.D., O.E., C.G., M.dL., R.B.D.: data interpretation; L.M., V.B., D.M., C.G., M.dL., R.B.D.: wrote a/o revised the manuscript. All authors reviewed the manuscript.

\section{Competing interests}

The authors declare no competing interests.

\section{Additional information}

Supplementary Information The online version contains supplementary material available at https://doi.org/ 10.1038/s41598-021-90084-y.

Correspondence and requests for materials should be addressed to L.M.

Reprints and permissions information is available at www.nature.com/reprints.

Publisher's note Springer Nature remains neutral with regard to jurisdictional claims in published maps and institutional affiliations. 
(c) (i) Open Access This article is licensed under a Creative Commons Attribution 4.0 International cc) License, which permits use, sharing, adaptation, distribution and reproduction in any medium or format, as long as you give appropriate credit to the original author(s) and the source, provide a link to the Creative Commons licence, and indicate if changes were made. The images or other third party material in this article are included in the article's Creative Commons licence, unless indicated otherwise in a credit line to the material. If material is not included in the article's Creative Commons licence and your intended use is not permitted by statutory regulation or exceeds the permitted use, you will need to obtain permission directly from the copyright holder. To view a copy of this licence, visit http://creativecommons.org/licenses/by/4.0/.

(C) The Author(s) 2021 